\title{
White sponge nevus presenting as genital lesions in a 28-year-old female
}

\begin{abstract}
A case is presented of white plaques occurring predominantly on the vulvar mucosa of a 28-year-old female diagnosed as white sponge nevus (WSN). WSN is a rare, autosomal dominant disorder involving mucous membranes. It predominantly affects the oral mucosa; however, it has been reported to rarely involve extraoral mucosal sites. In this case, histology and family history were key features leading to the correct diagnosis. WSN is an extremely rare cause of vulvar leukoplakia, yet it is important to recognize to allow for appropriate genetic counseling of this autosomal dominant disorder and to avoid misdiagnosis and the potential for subsequent exposure to ineffective treatment modalities.

Cutlan JE, Saunders N, Olsen SH and Fullen DR. White sponge nevus presenting as genital lesions in a 28 -year-old female. J Gutan Pathol 2010; 37: 386-389. (C) 2009 John Wiley \& Sons A/S.
\end{abstract}

\author{
J.E. Cutlan ${ }^{1}$, N. Saunders ${ }^{2}$, \\ S.H. Olsen ${ }^{1}$ and D.R. Fullen ${ }^{1}$ \\ ${ }^{1}$ Department of Pathology, and \\ ${ }^{2}$ Department of Obstetrics and Gynecology \\ University of Michigan, Ann Arbor, MI, USA
}

Douglas R. Fullen, MD. Department of Pathology University of Michigan

Tel: (734) $764-4460$

Fax: (734)764-4690

e-mail: dfullen@med.umich.edu

\section{Introduction}

White sponge nevus (WSN) is a rare, benign, autosomal dominant mucosal disorder that primarily affects the oral mucosa, though genital, laryngeal and esophageal mucosal involvement have also been documented. ${ }^{1-3}$ Mutations in the genes encoding mucosal specific keratins, K4 and K13, lead to the characteristic mucosal white plaques with a spongy clinical appearance. ${ }^{4,5}$ Oral lesions typically present in early childhood. Involvement of the buccal mucosa is commonly bilateral; similar changes may occur on the tongue, labial mucosa and floor of the mouth. The histologic features seen are acanthosis, parakeratosis, cytoplasmic clearing of spinous layer keratinocytes and characteristic perinuclear eosinophilic condensations that correspond to abnormal aggregation of cytokeratin tonofilaments. ${ }^{6}$ Herein we present a case of WSN presenting primarily as genital lesions in a previously healthy 28-year-old female.

\section{Case report}

The patient is a healthy 28-year-old Caucasian female with an extensive history of vaginal lesions since childhood. These lesions were presumed to be candidal vaginitis and she was treated, albeit unsuccessfully, with fluconazole at outside institutions. Upon presentation at the University of
Michigan Obstetrics and Gynecology Center, she complained of painful and pruritic vulvar lesions. In addition, the lesions would intermittently erode causing further pain, discomfort, and dyspareunia. Further investigation into the patient's family history revealed a history of WSN manifesting as oral lesions in her father and paternal uncle.

Physical examination revealed white plaques occurring on the genitalia and to a lesser extent on the oral mucosa (Fig. 1). Pelvic examination was remarkable for thick, white plaques present at the vestibule and extending into the introitus (Fig. 2). Speculum examination revealed otherwise normal vaginal mucosa. Biopsies from the edge of the lesion were sent for direct immunofluorescence and routine histologic evaluation. The initial clinical impression was lichen planus; thus, the patient was started on a high potency topical corticosteroid. A tissue culture for fungi was obtained and yielded no growth.

Histologic examination of the vulvar biopsy showed acanthosis, parakeratosis and focal spongiosis with neutrophilic inflammation (Fig. 3). Within the spinous layer, the keratinocytes had cytoplasmic clearing and perinuclear eosinophilic condensations. The perinuclear condensations are characteristic for WSN (Fig. 4). Owing to the neutrophilic infiltrate, a Periodic acid-Schiff with diastase stain (PAS-D) was performed to rule out superimposed fungal 


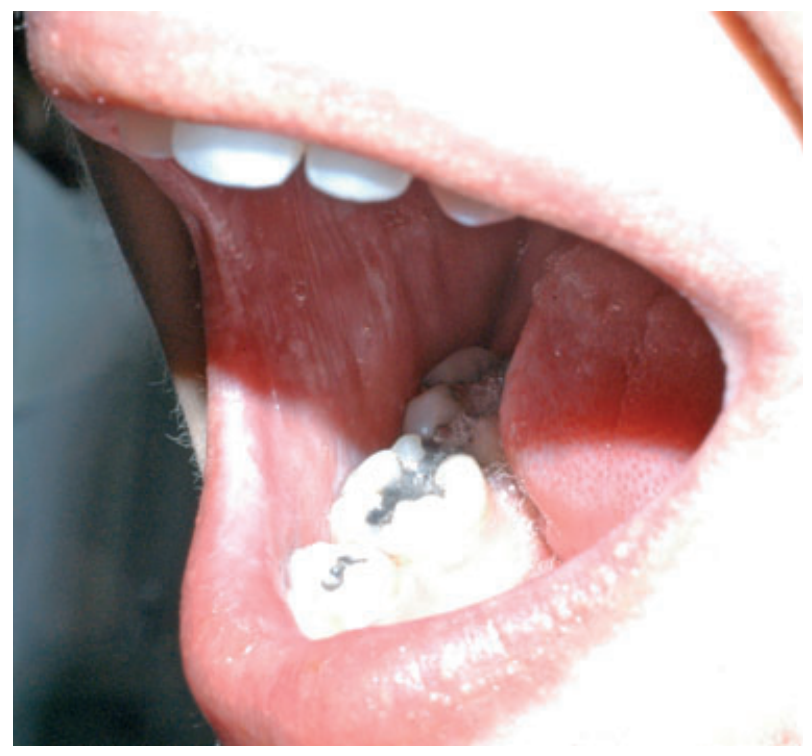

Fig. 1. White plaques involving the buccal mucosa.

infection and no fungal organisms were seen. Direct immunofluorescence examination of the vulva biopsy showed no specific immune deposits.

\section{Discussion}

The differential diagnosis for vulvar leukoplakia is vast, and includes inflammatory, premalignant/malignant, infectious and congenital abnormalities. With regard to inflammatory conditions causing leukoplakia, some of the most common etiologies include lichen sclerosus, lichen planus and lichen simplex chronicus. Based on the presence of pruritis and the clinical appearance of the lesions, lichen planus was initially suspected in our patient. Lichen planus is an inflammatory disorder of unknown etiology presenting as violaceous polygonal papules and plaques that typically affect the flexor surfaces of the extremities, although oral and genital involvement may also occur. In fact, in patients with oral lichen planus, concurrent vulvovaginal disease occurs in up to $19 \%$ of cases. ${ }^{7}$ When lichen planus involves the vulva, lesions typically appear as white reticulated areas with occasional surrounding erythema and/or erosions. Lichen planus produces a triad of histologic features that include hyperkeratosis, basal layer destruction, and a lichenoid infiltrate at the dermal-epidermal junction. The diagnosis may be complicated by lack of classic histology because of poorly formed lesions or co-existing secondary changes from topical applications, scratching or superimposed infection. The clinical picture in this case was fairly consistent with lichen planus involving the oral and genital mucosa; however, the histologic features in the biopsy did not support the

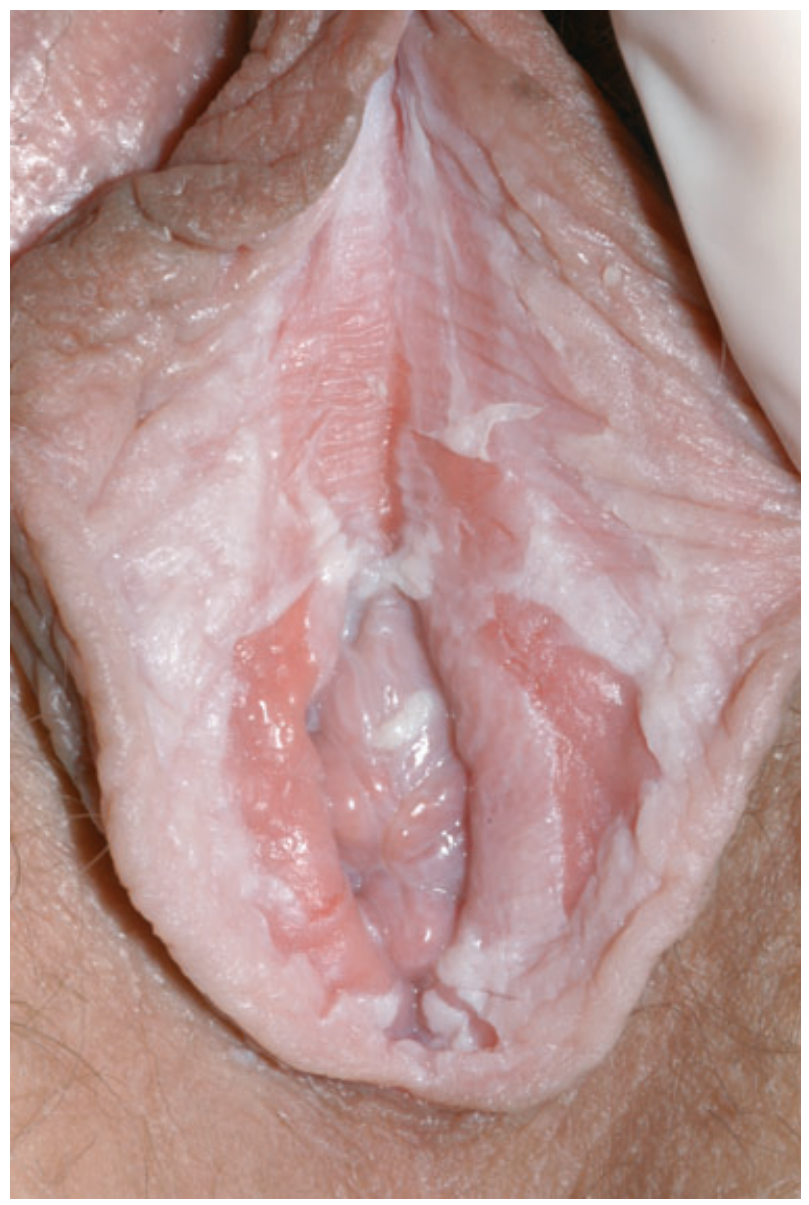

Fig. 2. Thick, white plaques involving the genitalia.

clinical diagnosis. Other common causes of vulvar leukoplakia include vulvar intraepithelial neoplasia and infections. In this case, a pre-malignant diagnosis was unlikely because vulvar intraepithelial neoplasia is most common in women greater than 60 years of age, and this patient's lesions had been present since childhood. Moreover, the biopsy lacked histologic evidence of nuclear crowding, cellular atypia and increased mitotic index seen in vulvar intraepithelial neoplasia. An infectious etiology, such as candidal vaginitis, was previously considered to be the most likely diagnosis clinically, but this patient shown no clinical improvement after multiple courses of fluconazole. In addition, no yeast spores or pseudohyphae were identified on routine histology or PAS-D staining, and a yeast culture yielded no growth.

Congenital diseases (genodermatoses) should also be considered in the differential diagnosis of leukoplakia, especially when the lesions are present in childhood. These genodermatoses include pachyonychia congenita (PC), dyskeratosis congenita (DC) and WSN. PG is an autosomal dominant inherited disorder of keratinization characterized clinically by a 


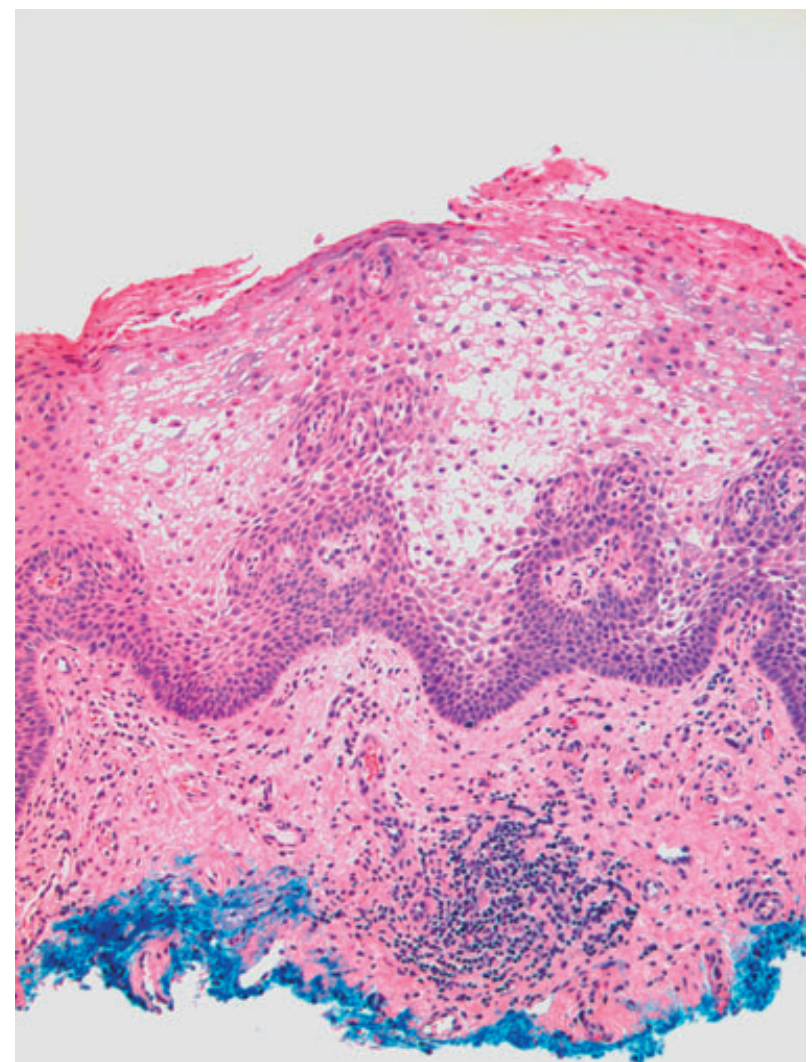

Fig. 3. Vulvar epithelium with acanthosis, parakeratosis and spongiosis. $(\mathrm{H} \& \mathrm{E}, 100 \times)$.

triad of subungunal hyperkeratosis, keratosis palmaris et plantaris and thickened white plaques involving the oral mucosa. The oral lesions of PC resemble WSN both clinically and histologically. Differentiation between these two entities requires evaluation for the additional dermatologic findings in the former. DG is most commonly an $\mathrm{X}$-linked recessive disorder of abnormal keratinization, although it can also be inherited in an autosomal dominant manner, thereby affecting both genders. Clinically, a triad of nail dystrophy, keratosis of oral and anal mucosa, and netlike pigmentation similar to poikiloderma atrophicans vasculare characterize the disorder. The previous congenital diseases may have oral findings similar to WSN; however, to our knowledge, no cases of $\mathrm{PC}$ or $\mathrm{DC}$ involving vulvovaginal mucosa have been reported to date. In contrast, WSN is a congenital mucosal disorder that, in addition to affecting the oral mucosa, may rarely involve other mucosal sites. It is an autosomal dominant inherited disorder with variable penetrance resulting in multiple family members being involved to varying degrees as was evident with this proband and her family. Recent studies have shown that mutations in the genes encoding mucosal specific keratins, K4 and K13, lead to the characteristic mucosal white plaques. Furthermore, a mouse

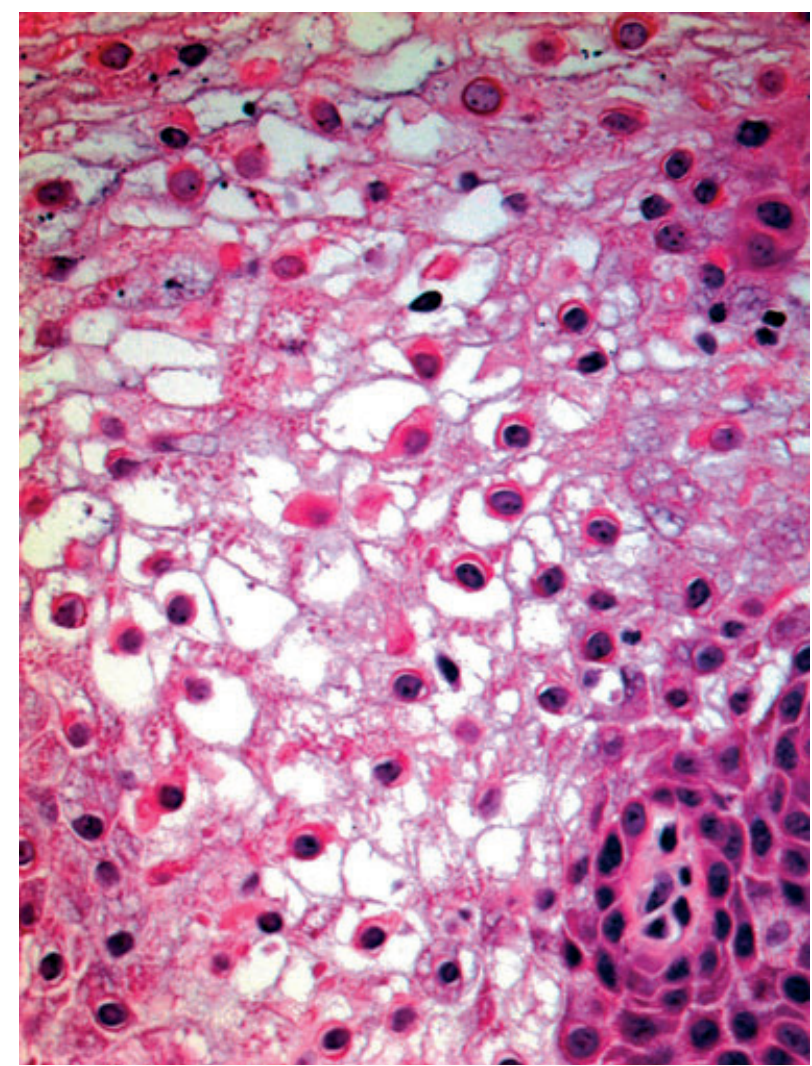

Fig. 4. Keratinocytes with extensive vacuolization and the characteristic perinuclear eosinophilic condensations of WSN (white sponge nevus). (H\&E, $400 \times)$.

model with a missense mutation in $\mathrm{K} 4$, recapitulates the findings of WSN. ${ }^{8}$ The resultant perinuclear eosinophilic condensations that are so characteristic of WSN are because of the abnormal coalescence of keratin tonofilaments within the cytoplasm of mucosal keratinocytes.

Rendering the correct diagnosis of leukoplakia is important for providing the most effective treatment and patient counseling. WSN has been known to be effectively treated with tetracycline or penicillin, ${ }^{9,10}$ whereas inflammatory conditions like lichen planus are normally treated with topical immunosuppressive therapy. In terms of premalignant/malignant disease, further excision or close follow up may be indicated for appropriate patient care. Antibiotics or antifungals may be needed to treat specific infectious etiologies.

In summary, we describe an unusual case of WSN presenting primarily with lesions in the genitalia resulting in clinical confusion with inflammatory causes of leukoplakia. Making the correct diagnosis enables the patient to receive appropriate genetic counseling of this autosomal dominant disorder and avoids misdiagnosis leading to ineffective treatment or potentially adverse side effects of unnecessary 


\section{White sponge nevus presenting as genital lesions}

medications and patient dissatisfaction over failure to achieve a therapeutic response. Clinical and family histories were helpful in contributing to the correct diagnosis of WSN in this patient; however, clinical history is not always provided to the pathologist evaluating the biopsy specimens. Thus, it is important to consider WSN in the differential diagnosis of vulvovaginal leukoplakia.

\section{References}

1. Lucchese A, Favia G. White sponge naevus with minimal clinical and histological changes: report of three cases. J Oral Pathol Med 2006; 35: 317.

2. Nichols GE, Cooper PH, Underwood PB Jr, Greer KE. White sponge nevus. Obstet Gynecol 1990; 76: 545.

3. Timmer R, Seldenrijk CA, Gorp LH, Dingemans KP, Bartelsman JF, Smout AJ. Esophageal white sponge nevus associated with severe dysphagia and odynophagia. Digest Dis Sci 1997; 42: 1914.
4. Terrinoni A, Rugg EL, Lane EB, et al. A novel mutation in the keratin 13 gene causing oral white sponge nevus. J Dent Res 2001; 80: 919.

5. Rugg EL, McLean WH, Allison WE, et al. A mutation in the mucosal keratin $\mathrm{K} 4$ is associated with oral white sponge nevus. Nat Genet 1995; 11: 450.

6. Morris R, Gansler TS, Rudisill MT, Neville B. White sponge nevus. Diagnosis by light microscopic and ultrastructural cytology. Acta Cytol 1988; 32: 357.

7. Rogers RS, Eisen D. Erosive oral lichen planus with genital lesions: the vulvovaginal-gingival syndrome and the penogingival syndrome. Dermatol Clin 2003; 21: 91.

8. McGowan K, Fuchs H, Hrabe de Angelis M, Barsh GS. Identification of a Keratin 4 mutation in a chemically induced mouse mutant that models white sponge nevus.J Invest Dermatol 2007; 127: 60.

9. Lamey PJ, Bolas A, Napier SS, Darwazeh AM, Macdonald DG. Oral white sponge naevus: response to antibiotic therapy. Clin Exp Dermatol 1998; 23: 59.

10. Alinovi A, Benoldi D, Pezzarossa E. White sponge nevus: successful treatment with penicillin. Acta Derm Venereol 1983; 63: 83. 